\title{
Indication and Usefulness of Bile Juice Cytology for Diagnosis of Gallbladder Cancer
}

\author{
Hiroshi Itsuki (D), ${ }^{1}$ Masahiro Serikawa, ${ }^{1}$ Tamito Sasaki, ${ }^{2}$ Yasutaka Ishii, ${ }^{1}$ Ken Tsushima, \\ Yoshinari Furukawa, ${ }^{3}$ Yoshiaki Murakami, ${ }^{4}$ Koji Arihiro, ${ }^{5}$ and Kazuaki Chayama ${ }^{1}$ \\ ${ }^{1}$ Department of Gastroenterology and Metabolism, Graduate School of Biomedical \& Health Sciences, Hiroshima University, \\ Hiroshima, Japan \\ ${ }^{2}$ Department of Gastroenterology, Hiroshima Prefectural Hospital, Hiroshima, Japan \\ ${ }^{3}$ Department of Gastroenterology, Hiroshima Red Cross Hospital \& Atomic-bomb Survivors, Hiroshima Red Cross Hospital \& \\ Atomic-bomb Survivors Hospital, Hiroshima, Japan \\ ${ }^{4}$ Department of Surgery, Applied Life Sciences Institute of Biomedical and Health Sciences, Hiroshima University, Hiroshima, Japan \\ ${ }^{5}$ Department of Anatomical Pathology, Hiroshima University Hospital, Hiroshima, Japan
}

Correspondence should be addressed to Hiroshi Itsuki; h.itsuki@hiroshima-med.jrc.or.jp

Received 24 August 2017; Revised 6 February 2018; Accepted 11 March 2018; Published 15 April 2018

Academic Editor: Atsushi Irisawa

Copyright (C) 2018 Hiroshi Itsuki et al. This is an open access article distributed under the Creative Commons Attribution License, which permits unrestricted use, distribution, and reproduction in any medium, provided the original work is properly cited.

\begin{abstract}
Aim. We examined the effectiveness of bile juice cytology for distinguishing between benign and malignant gallbladder lesions of the protruding type with various sampling points, sampling methods, and macroscopic forms in order to discuss the effectiveness of the endoscopic transpapillary gallbladder drainage (ETGD) cytology. Methods. We studied 162 cases of patients with a lesion localized within the gallbladder. At first, we examined the effectiveness for diagnosis of ETBD cytology using ERC and then that of the first ETGD cytology after placing the ETGD. Next, we examined the diagnostic effectiveness of the washed ETGD cytology by using the ETGD. Finally, we examined complications. Results. In the final diagnoses, we identified 33 cases of adenocarcinoma, 10 cases of adenoma, 63 cases of ADM, 35 cases of nonneoplastic polyp, and 21 cases of chronic cholecystitis. It was found that the sensitivity of ETBD cytology was $3.6 \%$ and that of ETGD cytology was $59.1 \%$. In the comparison of diagnostic effectiveness of cytologic diagnosis using samples of bile juice from the gallbladder collected by different methods, the sensitivities were $38.9 \%$ and $73.3 \%$ for the first and washed ETGD cytologies, respectively. In the comparison of the diagnostic effectiveness of gallbladder bile juice cytology using samples collected for different forms of lesion and by different methods, the sensitivities were $38.9 \%$ and $73.3 \%$, respectively, for the first and washed ETGD cytologies for flat gallbladder wall thickening, while it was impossible to diagnose for lesions of GB polyp. Conclusion. For diagnosis of gallbladder cancer, we consider that the ETGD cytology should be taken into consideration for lesions of flat gallbladder wall thickening, for which it is difficult to distinguish between benign and malignant lesions.
\end{abstract}

\section{Introduction}

Recent improvements in diagnostic imaging technology have revealed characteristics of gallbladder cancer in images [1, 2]. Also, diagnosis methods are now being organized systematically. However, there are still many clinical cases that are difficult to diagnose [3,4]. Gallbladder lesions of the protruding type are roughly classified based on their macroscopic forms into gallbladder polyps (GB polyps) and flat gallbladder wall thickenings. The cholesterol polyp is the type of GB polyp that is found most frequently. However, gallbladder adenomas and adenoma cancers are also found as GB polyps (Figures 1(a) and $1(\mathrm{~b})$ ). Some flat gallbladder wall thickenings are represented in images as sessile polyps as well as wall thickenings. They include early-stage cancer and advanced cancer that has advanced to the SS stage or further, in addition to nonneoplastic lesions of the adenomyomatosis (ADM) and xanthogranulomatous cholecystitis (XGC) (Figures 1(c) and $1(\mathrm{~d})$ ). Because the surgical resection rate for 


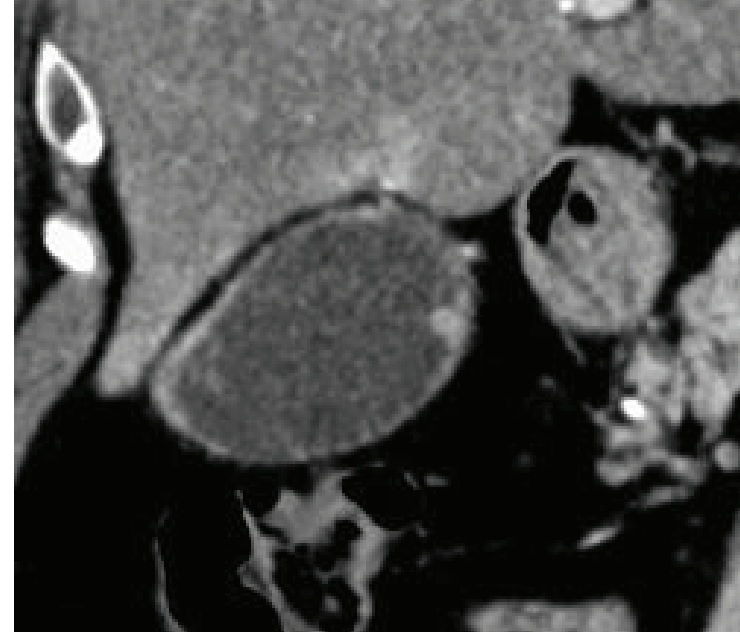

(a)

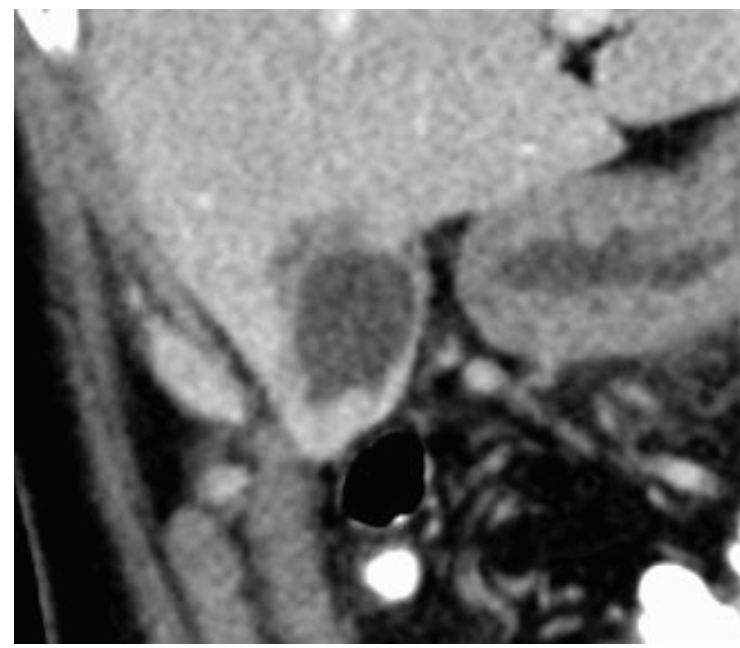

(c)

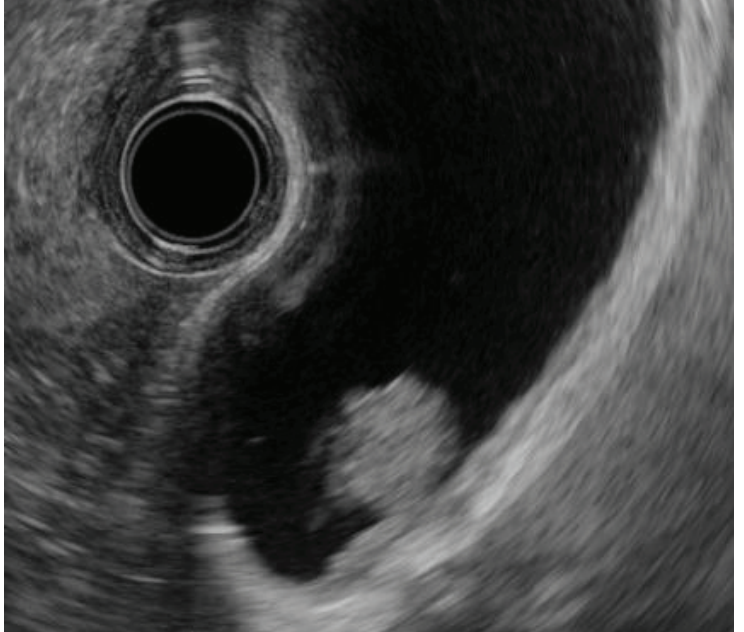

(b)

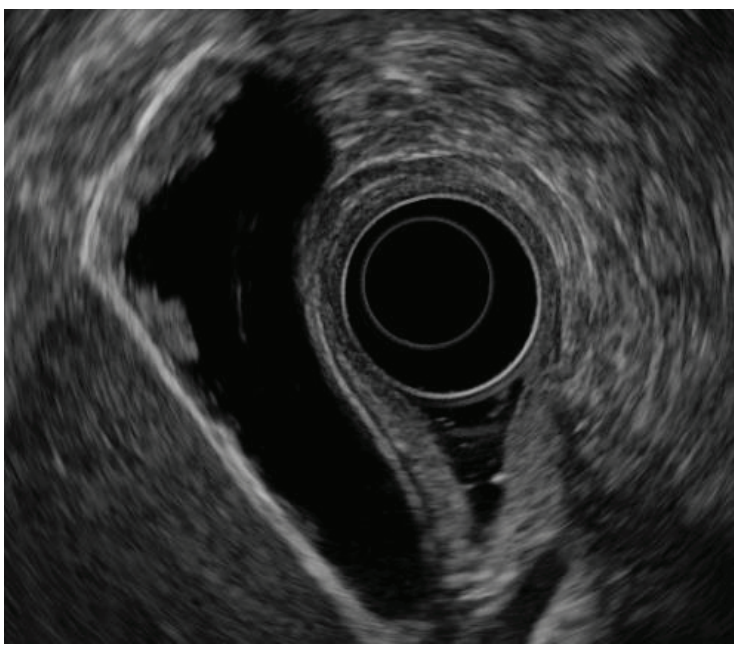

(d)

Figure 1: Forms of protruding type of gallbladder lesion. (a, b) A protrusion that exhibits a contrast effect is found in the gallbladder body in an abdominal CT scan image. It abruptly rises to show a pedunculated form. Classified to be a GB polyp based on the EUS image. The site was at the bottom of the gallbladder and the invasion depth was Tis, and it was a case of T1N0M0 Stage I. (c, d) Localized wall thickening that exhibits a contrast effect is found at the bottom of the gallbladder in an abdominal CT scan image. Classified to be a flat gallbladder wall thickening based on the EUS image. The site was at the bottom of the gallbladder and the invasion depth was ss, and it was a case of T2N1M0 Stage IIIb.

gallbladder cancers is not exactly high, and considering the prognosis, it is required to diagnose it in its early stages [5-7]. Pathological diagnosis using bile juice cytology is an important method because it plays a significant role in definitive differential diagnosis between benign and malignant tumors and determination of the treatment method. In particular, it was recently reported that ETGD cytology using bile juice collected by endoscopic transpapillary gallbladder drainage (ETGD) is useful for diagnosis $[3,4]$. However, there are still a large number of unresolved issues that must be resolved regarding its application and interpretation.

In this study, we examined the diagnostic effectiveness of bile juice cytology for differential diagnosis between benign and malignant gallbladder lesions of the protruding type with various sampling points, sampling methods, and macroscopic forms in order to discuss the application and effectiveness of the ETGD cytology.

\section{Patients and Method}

2.1. Patients and Protocol. We studied 162 cases of patients who were examined in our clinics and whose lesions were localized within the gallbladder. Among these cases, surgical resection and histopathological diagnosis were conducted in 102 cases and follow-up observation was conducted in the remaining 60 cases. For all the cases, the lesions were classified based on their forms, as identified in endoscopic ultrasonography (EUS), and then bile juice was collected by a transpapillary method under ERC. 


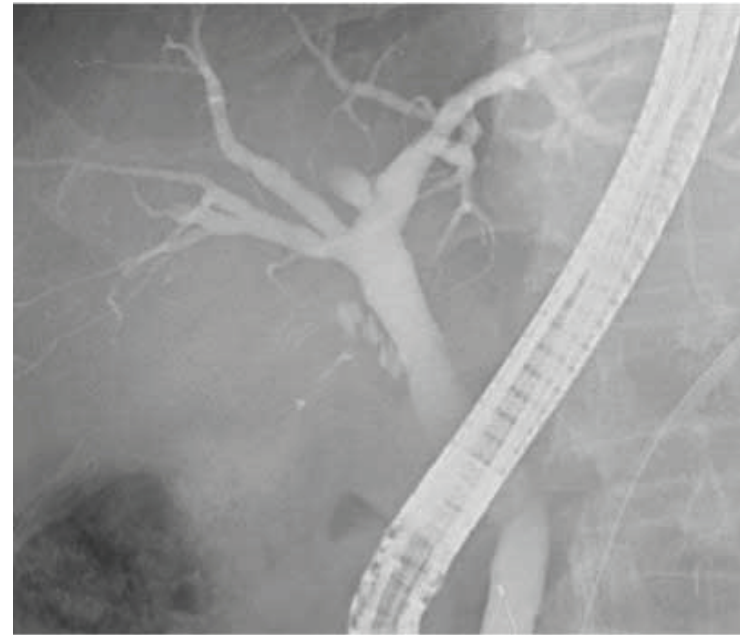

(a)

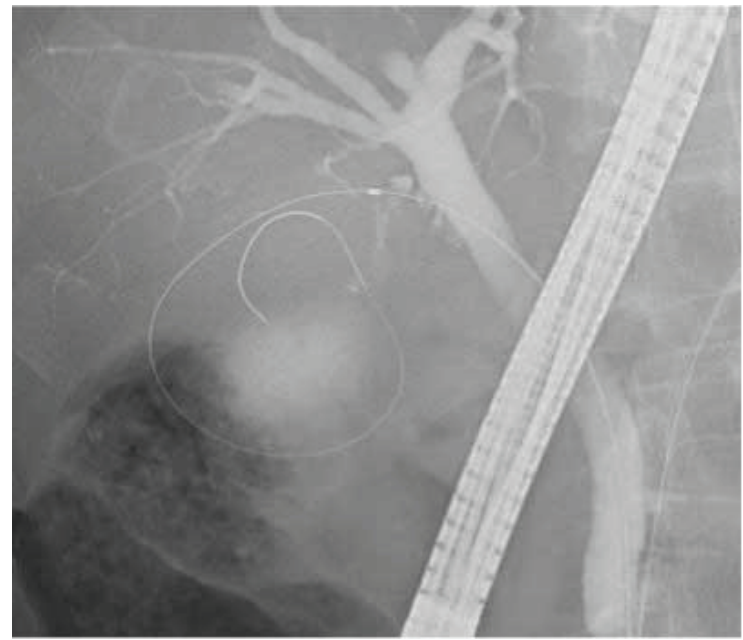

(c)

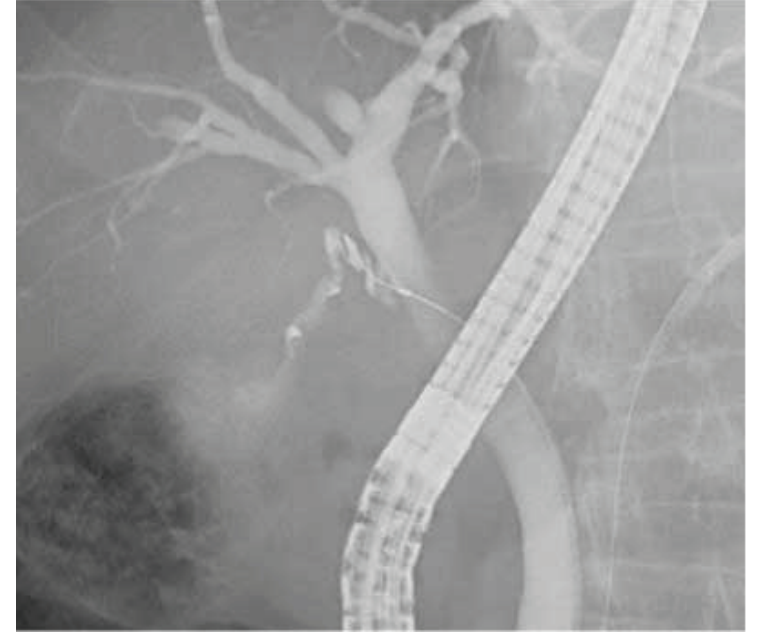

(b)

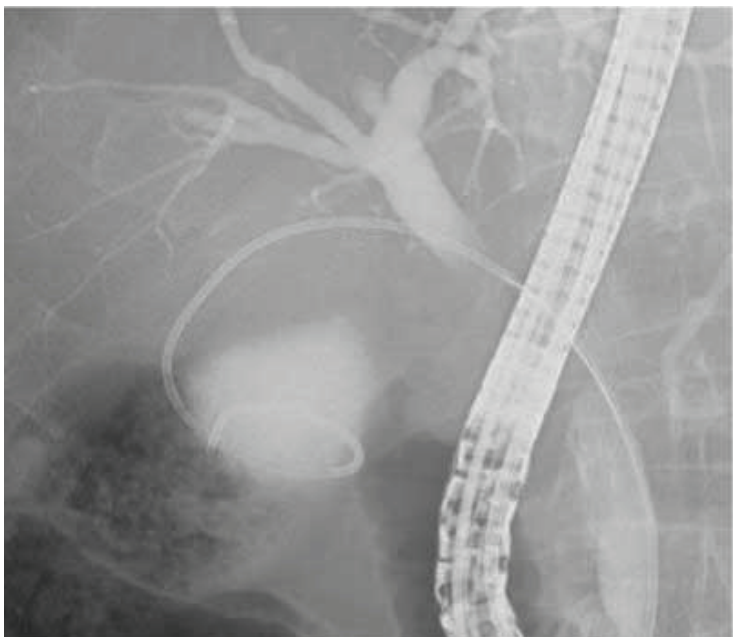

(d)

FIgURE 2: ETGD placement method. (a) Contrast is increased to enable location of the branching point between the bile duct and the cystic duct. (b) The tip of the guiding wire is advanced into the cystic duct. (c) The guiding wire is turned at least one rotation in the gallbladder. (d) A pernasal bile drainage tube of $5 \mathrm{Fr}$ pigtail type is put in place.

2.2. Procedure for Sampling Bile. Bile juice from the bile duct and bile juice from the gallbladder were collected as the sample for cytologic examination under endoscopic retrograde cholangiography (ERC). For the bile juice from the bile duct, a sufficient amount was siphoned through the imaging catheter after deep intubation into the bile duct. For the bile juice from the gallbladder, a guiding wire of 0.025 -inch diameter with an angled tip was inserted into the gallbladder under ERC while carefully seeking the inside of the cystic duct. The guiding wire was turned at least one rotation, and a pernasal bile drainage tube of $5 \mathrm{Fr}$ pigtail type (Flexima Nasobiliary Catheter, Boston Scientific Corporation) was placed in the gallbladder (Figures 2). The bile juice from the gallbladder was first siphoned and collected for cytologic examination just after the ETGD was placed (first ETGD cytology). The next day, the inside of the gallbladder was washed with normal saline solution using the ETGD, and the collected sample of wash solution was used for the cytologic examination (washed ETGD cytology). When a lot of debris was found in the sample of wash solution, the inside of the gallbladder was further washed sufficiently to siphon off and remove the debris.

\section{Results}

3.1. Classification of Cases. In the final diagnoses, we identified 33 cases of adenocarcinoma, 10 cases of adenoma, 63 cases of ADM, 35 cases of nonneoplastic polyp, and 21 cases of chronic cholecystitis. In terms of the classification of form, 43 and 119 cases were classified as GB polyp and flat gallbladder wall thickening, respectively. The cases of GB polyp consisted of 5 cases of adenocarcinoma, 10 cases of adenoma, and 28 cases of nonneoplastic polyp. All the adenocarcinomata were carcinomas in adenoma. The cases of flat gallbladder wall thickening consisted of 28 cases of adenocarcinoma, 63 cases of ADM, 7 cases of nonneoplastic lesion, and 21 cases of chronic cholecystitis (Table 1). 
TABLE 1: Details of protruding type of gallbladder lesion.

\begin{tabular}{lcc}
\hline & GB polyp & Flat gallbladder wall thickening \\
\hline Adenocarcinoma & 5 & 28 \\
Adenoma & 10 & 0 \\
Adenomyosis & 0 & 63 \\
Nonneoplastic polyp & 28 & 7 \\
Chronic cholecystitis & 0 & 21 \\
Total & 43 & 119 \\
\hline
\end{tabular}

TABLE 2: Diagnostic effectiveness comparison for ETBD cytology and ETGD cytology.

\begin{tabular}{lcc}
\hline & $\begin{array}{c}\text { Bile duct bile juice } \\
(n=137)\end{array}$ & $\begin{array}{c}\text { Gallbladder bile juice } \\
(n=133)\end{array}$ \\
\hline Sensitivity & $3.6 \%$ & $59.1 \%$ \\
Specificity & $100 \%$ & $100 \%$ \\
Accuracy & $80.1 \%$ & $93.2 \%$ \\
PPV & $100 \%$ & $100 \%$ \\
NPV & $80.2 \%$ & $92.5 \%$ \\
\hline
\end{tabular}

3.2. Comparison of Diagnostic Effectiveness for Sampling Point of Bile Juice. At first, we examined the diagnostic effectiveness of bile juice cytology with various sampling points. For the diagnostic effectiveness of ETBD cytology, we found $3.6 \%$ sensitivity, $100 \%$ specificity, $80 \%$ accuracy, $100 \%$ positive predictive value (PPV), and $80.2 \%$ negative predictive value (NPV). Next, we examined the diagnostic effectiveness of ETGD cytology. For the diagnostic effectiveness of ETGD cytology, we found 59.1\% sensitivity, $100 \%$ specificity, $93.2 \%$ accuracy, 100\% PPV, and $92.5 \%$ NPV. As such, the results for ETGD cytology were better than those for ETBD cytology (Table 2).

3.3. Comparison of Diagnostic Effectiveness for Sampling Method for Bile Juice from the Gallbladder. Next, we compared the diagnostic effectiveness of cytologic diagnosis using samples of bile juice from the gallbladder collected by different methods. For the first ETGD cytology using samples collected just after the ETGD was placed, we found $38.9 \%$ sensitivity, $100 \%$ specificity, $88.7 \%$ accuracy, $100 \% \mathrm{PPV}$, and $87.8 \% \mathrm{NPV}$. On the other hand, for the washed ETGD cytology using samples collected one day after the ETGD was placed, we found $73.3 \%$ sensitivity, $100 \%$ specificity, $95.4 \%$ accuracy, $100 \%$ PPV, and $94.8 \%$ NPV. The sensitivity and accuracy were improved in the washed ETGD cytology (Table 3).

3.4. Comparison of Diagnosability of Lesion Form in the First and Washed ETGD Cytologies. Finally, we examined the diagnostic effectiveness of the first and washed ETGD cytologies for different forms of the lesion. In the examination of bile juice from the gallbladder for GB polyp, no case was diagnosed as malignant for either the first or the washed ETGD cytology (data not shown). On the other hand, for flat gallbladder wall thickening, the ETGD cytology indicated 50\% sensitivity, $100 \%$ specificity, $88.2 \%$
TABLE 3: Diagnostic effectiveness comparison for first and washed ETGD cytologies.

\begin{tabular}{lcc}
\hline & $\begin{array}{c}\text { First ETGD cytology } \\
(n=97)\end{array}$ & $\begin{array}{c}\text { Washed ETGD cytology } \\
(n=88)\end{array}$ \\
\hline Sensitivity & $38.9 \%$ & $73.3 \%$ \\
Specificity & $100 \%$ & $100 \%$ \\
Accuracy & $88.7 \%$ & $95.4 \%$ \\
PPV & $100 \%$ & $100 \%$ \\
NPV & $87.8 \%$ & $94.8 \%$ \\
\hline
\end{tabular}

TABLE 4: Diagnostic effectiveness of ETGD cytology for forms of lesion.

\begin{tabular}{lcc}
\hline & $\begin{array}{c}\text { GB polyp } \\
(n=43)\end{array}$ & $\begin{array}{c}\text { Flat gallbladder wall thickening } \\
(n=119)\end{array}$ \\
\hline Sensitivity & $0.0 \%$ & $50.0 \%$ \\
Specificity & $100 \%$ & $100 \%$ \\
Accuracy & $88.4 \%$ & $88.2 \%$ \\
PPV & $0.0 \%$ & $100 \%$ \\
NPV & $88.4 \%$ & $86.7 \%$ \\
\hline
\end{tabular}

accuracy, 100\% PPV, and $86.7 \%$ NPV. The sensitivity varied based on the form of lesion (Table 4).

3.5. Accidental Symptoms Caused by ETGD Placement. Among the 162 cases we examined in this study, we identified an accidental symptom in 14 cases in total (8.6\%). These consisted of 1 case of obstructive jaundice, 3 cases of acute cholecystitis, 9 cases of acute pancreatitis, and 1 case of gallbladder perforation.

\section{Discussion}

Diagnosability of gallbladder cancer has been improved recently thanks to the improvements in ultrasonography (US) and EUS and their use in combination with multidetector-row computed tomography (MDCT) and magnetic resonance imaging (MRI) systems [8, 9]. Although findings from the examination of these images are of great importance in the diagnosis of gallbladder cancers, they include cases in which it is difficult to distinguish gallbladder cancers such as XGC and ADM, and unnecessarily large-scale operations are in some cases conducted $[3,10,11]$. Therefore, pathological diagnosis using bile juice cytology is an important method because it plays a significant role in the definitive differential diagnosis between benign and malignant tumors and the determination of treatment method.

The ETGD placement method was first conducted by Kozarek [12] in 1984, and it is said to be effective and safe for cases in which percutaneous drainage is difficult to conduct due to high risk of bleeding or acute cholecystitis with ascites [13]. Itoi et al. reported that the success rate of ETGD placement for gallbladder diseases was $81 \%$. For 162 cases in which we tried to perform selective intubation into the gallbladder, the success rate of selective 
insertion of the guiding wire was $80.9 \%$, which indicates almost the same level. It was possible to place the drainage tube in all the cases in which the guiding wire was successfully inserted to the gallbladder. Itoi et al. pointed out some factors for successful ETGD placement for gallbladder diseases, such as accurate determination of the location of the branching point between the bile duct and the cystic duct, coordinated handling of the cannula and the guiding wire for the spirally winding cystic duct, and use of a swing catheter tip for cases in which the cystic duct branches downward from the bile duct [14]. Many examinations have been conducted for the treatment of acute pancreatitis using the ETGD that is placed in the gallbladder [15]. On the other hand, only a few studies have been reported for diagnosis of gallbladder cancer based on ETGD cytology using the ETGD placed in the gallbladder to collect the sample.

In this study, we examined the effectiveness of bile juice cytology for differential diagnosis between benign and malignant gallbladder lesions of the protruding type. In the comparison of diagnostic effectiveness of various sampling points for bile juice, we found that the sensitivity of ETGD cytology increased to $59.1 \%$ from $3.6 \%$ for ETBD cytology. Naito et al. also reported that ETGD cytology showed a superior sensitivity of $78 \%$ for gallbladder cancer compared to $20 \%$ sensitivity for ETBD cytology [16]. Based on these results, it was concluded that the sample should be collected at a point near the lesion, inside the gallbladder, for differential diagnosis between benign and malignant gallbladder tumors.

Then we examined the sensibility of ETGD cytology for different macroscopic forms of lesion. The sensitivity for flat gallbladder wall thickening was $50 \%$, while the sensitivity for GB polyp was $0 \%$. In addition to that, we compared diagnostic effectiveness of sampling methods for bile juice from the gallbladder for flat gallbladder wall thickening. While the sensitivity in the first ETGD cytology for flat gallbladder wall thickening was $38.9 \%$, the sensitivity of the washed ETGD cytology was improved to $73.3 \%$. Tamada et al. also reported that the diagnostic effectiveness of cytologic diagnosis for gallbladder cancer using the first ETGD cytology was low and was improved by using the washed ETGD cytology while the ETGD was in place [15]. It is assumed that this is because the sample collected for the first ETGD cytology contains a lot of inflammatory cells and biliary sludge, and the epithelial cells of the gallbladder are altered. In the washed ETGD cytology, on the other hand, fresh exfoliated cells are collected due to the washing process. Matsubayashi et al. [17] reported that the washed ETGD cytology using ETGD was very effective for a case in which a malignant black tumor had spread into the stroma of the mucosa of the gallbladder. In this study, it was found that the cytologic diagnosis method is not capable of identifying gallbladder cancer of the GB polyp type, regardless of whether the sample is collected in the bile duct or the gallbladder. It was concluded that this was because all the malignant lesions of the GB polyp type were adenocarcinomata, and the volume of the tumor that constitutes the malignant lesion was rather small.
Considering the results described above, it was concluded that, considering the risk of complications, ERC is not required in the case of a GB polyp which is suspected to be a malignant lesion based on the image, and laparoscopic cholecystectomy should be performed immediately. However, needless to say, it is important to observe the lesion in detail using EUS since some lesions concurrently contain GB polyp and flat gallbladder wall thickening. On the other hand, it is thought that the effectiveness of the ETGD cytology for diagnosis of gallbladder cancer in flat gallbladder wall thickening is very high, particularly for the washed ETGD cytology using ETGD. Therefore, for diagnosis of flat gallbladder wall thickening, which is suspected to be gallbladder cancer, it is required to consider differential diagnosis between benign and malignant lesions using the ETGD cytology, in addition to various imaging diagnostic methods, in order to carefully determine the surgical procedure to be adopted.

When placing the ETGD, it is required to take the utmost care to avoid complications. Itoi et al. reported that they experienced a complication in $8.2 \%$ of 330 cases in which the ETGD was placed. They reported 2 cases of gallbladder perforation, 2 cases of bile leak, 1 case of bleeding, and 22 cases of the other complications. Among the 162 cases we examined in this study, we identified an accidental symptom in 14 cases in total (8.6\%). These consisted of 1 case of obstructive jaundice, 3 cases of acute cholecystitis, 9 cases of acute pancreatitis, and 1 case of gallbladder perforation. However, we did not experience any fatal complication, and all patients recovered after removal of the tube and/or conservative treatment. For the placement of ETGD, Mori et al. described that selective cannulation into the bile duct and the appropriate handling of the guiding wire are required, because a serious complication, such as pancreatitis or gallbladder perforation, can be caused after the ERCP [18].

We have described the results of our study on the effectiveness of bile juice cytology for differential diagnosis between benign and malignant gallbladder lesions of the protruding type. Needless to say, imaging diagnosis methods take a central role in the diagnosis of gallbladder cancer. However, we take the view that the ETGD cytology should be positively taken into consideration for lesions of flat gallbladder wall thickening for which it is difficult to distinguish between benign and malignant lesions and to determine the treatment policy, particularly the surgical procedure, to be adopted.

\section{Conflicts of Interest}

The authors declare that they have no conflicts of interest.

\section{References}

[1] S. J. Kim, J. M. Lee, J. Y. Lee et al., "Accuracy of preoperative Tstaging of gallbladder carcinoma using MDCT," American Journal of Roentgenology, vol. 190, no. 1, pp. 74-80, 2008.

[2] J. S. Lee, J. H. Kim, Y. J. Kim et al., "Diagnostic accuracy of transabdominal high-resolution US for staging gallbladder cancer and differential diagnosis of neoplastic polyps 
compared with EUS," European Radiology, vol. 27, no. 7, pp. 3097-3103, 2017.

[3] H. Suzuki, S. Wada, K. Araki et al., "Xanthogranulomatous cholecystitis: difficulty in differentiating from gallbladder cancer," World Journal of Gastroenterology, vol. 21, no. 35, pp. 10166-10173, 2015.

[4] S. H. Bang, J. Y. Lee, H. Woo et al., "Differentiating between adenomyomatosis and gallbladder cancer: revisiting a comparative study of high-resolution ultrasound, multidetector CT, and MR imaging," Korean Journal of Radiology, vol. 15, no. 2, pp. 226-234, 2014.

[5] Y. Ome, K. Hashida, M. Yokota, Y. Nagahisa, M. Okabe, and K. Kawamoto, "Laparoscopic approach to suspected T1 and T2 gallbladder carcinoma," World Journal of Gastroenterology, vol. 23, no. 14, pp. 2556-2565, 2017.

[6] K. Kai, H. Irie, T. Ide et al., "Actual status of clinical diagnosis in patients with primary gallbladder cancer associated with adenomyomatosis," Indian Journal of Gastroenterology, vol. 32, no. 6, pp. 386-391, 2013.

[7] R. P. Kiran, N. Pokala, and S. J. Dudrick, "Incidence pattern and survival for gallbladder cancer over three decades-an analysis of 10301 patients," Annals of Surgical Oncology, vol. 14, no. 2, pp. 827-832, 2007.

[8] G. Leem, M. J. Chung, J. Y. Park et al., "Clinical value of contrast-enhanced harmonic endoscopic ultrasonography in the differential diagnosis of pancreatic and gallbladder masses," Clinical Endoscopy, vol. 51, no. 1, pp. 80-88, 2017.

[9] S. J. Kim, J. M. Lee, J. Y. Lee et al., “Analysis of enhancement pattern of flat gallbladder wall thickening on MDCT to differentiate gallbladder cancer from cholecystitis," American Journal of Roentenology, vol. 191, no. 3, pp. 765-771, 2008.

[10] A. Y. Hammad, J. T. Miura, K. K. Turaga, F. M. Johnston, M. D. Hohenwalter, and T. C. Gamblin, "A literature review of radiological findings to guide the diagnosis of gallbladder adenomyomatosis," HPB, vol. 18, no. 2, pp. 129-135, 2016.

[11] B. J. Chang, S. H. Kim, H. Y. Park et al., "Distinguishing xanthogranulomatous cholecystitis from the wall-thickening type of early-stage gallbladder cancer," Gut and Liver, vol. 4, no. 4, pp. 518-523, 2010.

[12] R. A. Kozarek, "Selective cannulation of the cystic duct at time of ERCP," Journal of Clinical Gastroenterology, vol. 6, no. 1, pp. 37-40, 1984.

[13] M. J. Yang, B. M. Yoo, J. H. Kim et al., "Endoscopic nasogallbladder drainage versus gallbladder stenting before cholecystectomy in patients with acute cholecystitis and a high suspicion of choledocholithiasis: a prospective randomised preliminary study," Scandinavian Journal of Gastroenterology, vol. 51, no. 4, pp. 472-478, 2016.

[14] T. Itoi, A. Sofuni, F. Itokawa et al., "Preoperative diagnosis and management of thick-walled gallbladder based on bile cytology obtained by endoscopic transpapillary gallbladder drainage tube," Gastrointestinal Endoscopy, vol. 64, no. 4, pp. 512519, 2006.

[15] K. Tamada, H. Seki, K. Sato et al., "Efficacy of endoscopic retrograde cholecystoendoprosthesis (ERCCE) for cholecystitis," Endoscopy, vol. 23, no. 1, pp. 2-3, 1991.

[16] Y. Naito, Y. Okabe, A. Kawahara et al., "Usefulness of lavage cytology during endoscopic transpapillary catheterization into the gallbladder in the cytological diagnosis of gallbladder disease," Diagnostic Cytopathology, vol. 37, no. 6, pp. 402-406, 2009.
[17] H. Matsubayashi, Y. Kiyohara, K. Sasaki et al., "Metastatic malignant melanoma of the gallbladder diagnosed by cytology of endoscopic naso-gallbladder drainage fluid," Journal of Digestive Diseases, vol. 13, no. 3, pp. 190-194, 2012.

[18] Y. Mori, T. Itoi, T. H. Baron et al., “Tokyo guidelines 2018: management strategies for gallbladder drainage in patients with acute cholecystitis (with videos)," Journal of HepatoBiliary-Pancreatic Sciences, vol. 25, no. 1, pp. 87-95, 2018. 


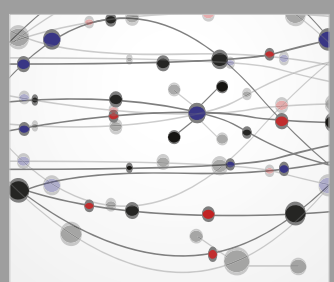

The Scientific World Journal
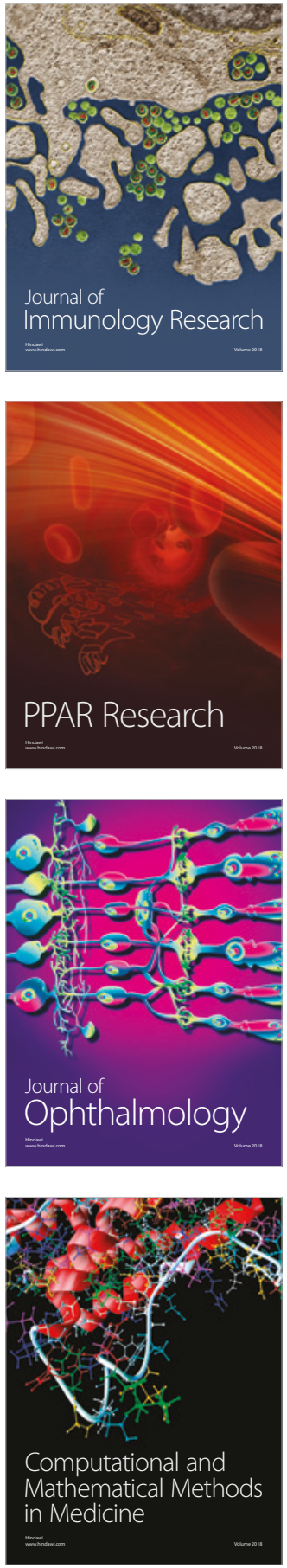

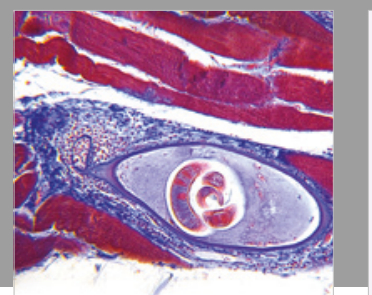

Gastroenterology Research and Practice

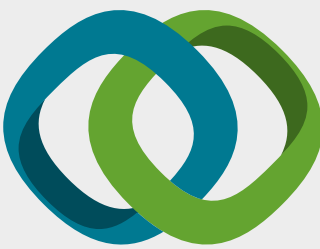

\section{Hindawi}

Submit your manuscripts at

www.hindawi.com
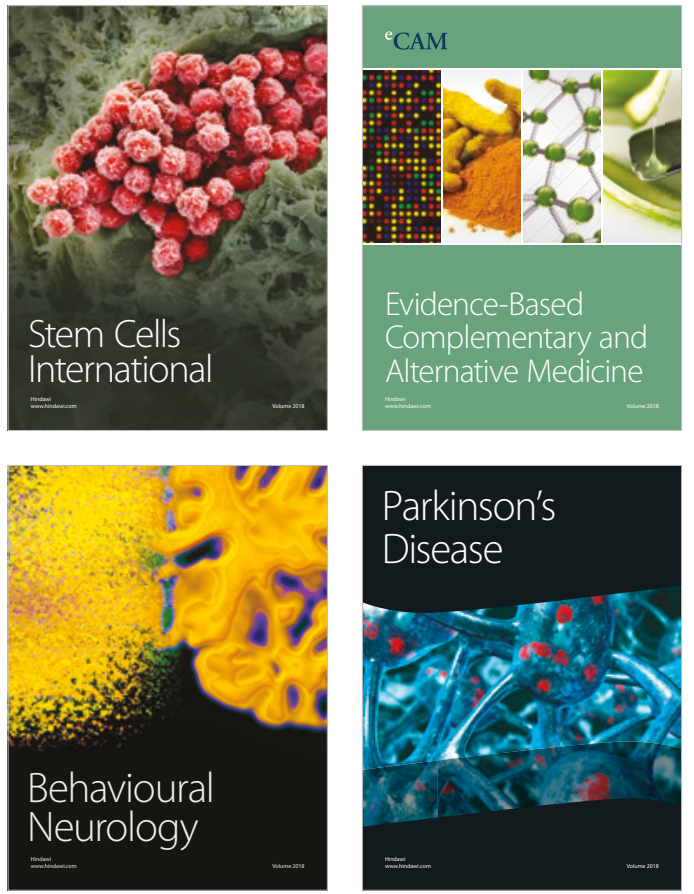

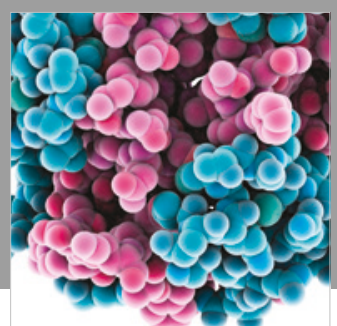

ournal of

Diabetes Research

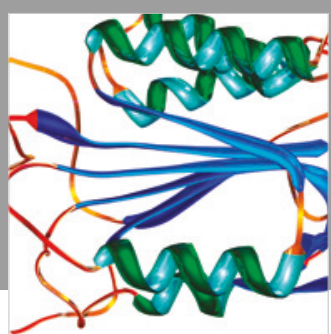

Disease Markers
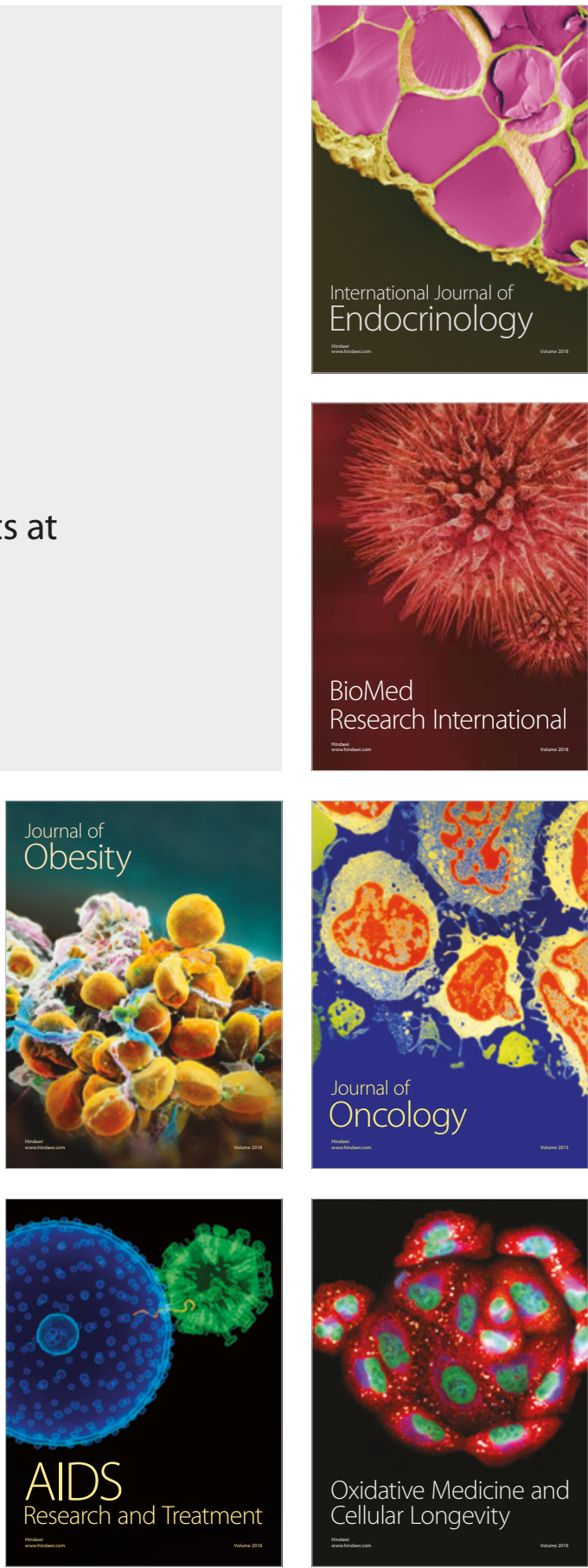